Pacific Journal of Mathematics

ARENA REGULARITY AND DISCRETE GROUPS 


\section{ARENS REGULARITY AND DISCRETE GROUPS}

\section{BRIAN FORREST}

Let $G$ be a locally compact group. Let $A_{p}(G)$ be the Herz algebra of $G$ associated with $1<p<\infty$. We show that if $A_{p}(G)$ is Arens regular, then $G$ is discrete. We also exhibit a number of sufficient conditions for such a group to be finite.

1. Introduction. Let $G$ be a locally compact group. For $1<p<$ $\infty$, let $A_{p}(G)$ denote the linear subspace of $C_{0}(G)$ consisting of all functions of the form $u(x)=\sum_{i=1}^{\infty}\left(f_{i} * \tilde{g}_{i}\right)^{\vee}$, where $f_{i} \in L_{p}(G)$, $g_{i} \in L_{q}(G), \frac{1}{p}+\frac{1}{q}=1, \sum_{i=1}^{\infty}\|f\|_{p}\|g\|_{q}<\infty, f^{\vee}(x)=f\left(x^{-1}\right)$ and $\tilde{f}(x)=\overline{f\left(x^{-1}\right)} . A_{p}(G)$ is a commutative Banach algebra with respect to pointwise multiplication and the norm

$$
\|u\|_{A_{p}(G)}=\inf \left\{\sum_{i=1}^{\infty}\left\|f_{i}\right\|_{p}\left\|g_{i}\right\|_{q} \mid u(x)=\sum_{i=1}^{\infty}\left(f_{i} * \tilde{g}_{i}\right)^{\vee}\right\} .
$$

When $p=2, A_{2}(G)$ is the Fourier algebra of $G$ as introduced by Eymard in [7]. For general $p$, the algebras $A_{p}(G)$ were introduced and first studied by Herz [13].

In this paper we will study the structure of the second dual $A_{p}(G)^{* *}$ as a Banach algebra with respect to the two Arens products. In particular, we will show that if $A_{p}(G)$ is Arens regular, then $G$ is discrete. When $p=2$, we show that for a large class of groups, Arens regularity will imply finiteness.

2. Preliminaries. Let $G$ be a locally compact group with a fixed left Haar measure $\lambda$. For $1 \leq p \leq \infty$, let $L_{p}(G)$ be the usual Banach space of equivalence classes of $p$-integrable (or essentially bounded) functions on $G$. The algebras $A_{p}(G)$ for $1<p<\infty$ will be as defined in $\S 1$. When $p=2$ we will write $A(G)$ for $A_{2}(G)$.

For $1<p<\infty$, let $P F_{p}(G)$ and $P M_{p}(G)$ denote the closure of $L_{1}(G)$, considered as an algebra of convolution operators on $L_{p}(G)$, with respect to the norm topology and the weak operator topology respectively in $\mathscr{B}\left(L_{p}(G)\right)$, the bounded operators on $L_{p}(G)$. The space $P M_{p}(G)$ can be identified with the dual of $A_{p}(G)$ for each $1<p<\infty$ [see 19, p. 94]. 
Let $B_{p}(G)$ denote the space of multipliers of $A_{p}(G)$. Then $B_{p}(G)$ with the norm

$$
\|u\|_{B_{p}(G)}=\sup \left\{\|u v\|_{A_{p}(G)} \mid v \in A_{p}(G),\|v\|_{A_{p}(G)} \leq 1\right\}
$$

is a commutative Banach algebra with respect to pointwise multiplication.

Let $A \subseteq G$ be closed. We will denote by $I_{p}(A)$ the closed ideal of $A_{p}(G)$ of the form $\left\{u \in A_{p}(G) \mid u(x)=0\right.$ for every $\left.x \in A\right\}$. Given an ideal $I \subseteq A_{p}(G)$, we denote by $Z(I)$ the set $\{x \in G \mid u(x)=0$ for every $u \in I\}$.

Let $\mathscr{A}$ be a Banach algebra. Then $\mathscr{A}^{* *}$ can be be given two multiplications which extend the multiplication of $\mathscr{A}$ and for which $\mathscr{A}^{* *}$ becomes a Banach algebra. These products were introduced by Arens in [1]. They are defined as follows:

(1a) $\langle u \cdot T, v\rangle=\langle T, v u\rangle$ for every $u, v \in \mathscr{A}, T \in \mathscr{A}^{* *}$,

(1b) $\langle T \odot \Gamma, u\rangle=\langle\Gamma, u \cdot T\rangle$ for every $u \in \mathscr{A}, T \in \mathscr{A}^{*}, \Gamma \in \mathscr{A}^{* *}$,

(1c) $\left\langle\Gamma_{1} \odot \Gamma_{2}, T\right\rangle=\left\langle\Gamma_{2}, T \odot \Gamma_{1}\right\rangle$ for every $T \in \mathscr{A}^{*}, \Gamma_{1}, \Gamma_{2} \in \mathscr{A}^{* *}$,

(2a) $\langle T \square u, v\rangle=\langle T, u v\rangle$ for every $u, v \in \mathscr{A}, T \in \mathscr{A}^{*}$,

(2b) $\langle\Gamma \square T, u\rangle=\langle\Gamma, T \square u\rangle$ for every $u \in \mathscr{A}, T \in \mathscr{A}^{*}, \Gamma \in \mathscr{A}^{* *}$,

(2c) $\left\langle\Gamma_{1} \square \Gamma_{2}, T\right\rangle=\left\langle\Gamma_{1}, \Gamma_{2} \square T\right\rangle$ for every $T \in \mathscr{A}^{*}, \Gamma_{1}, \Gamma_{2} \in \mathscr{A}^{* *}$.

In general, $\Gamma_{1} \odot \Gamma_{2}=\Gamma_{2} \square \Gamma_{2}$ may fail for some $\Gamma_{1}, \Gamma_{2} \in \mathscr{A}^{* *}$. If $\Gamma_{1} \odot \Gamma_{2}=\Gamma_{1} \square \Gamma_{2}$ for every $\Gamma_{1}, \Gamma_{2} \in \mathscr{A}$, then $\mathscr{A}$ is said to be Arens regular.

Let $\mathscr{A}$ be a commutative Banach algebra. Then $u \cdot T=T \square u$. Hence $\mathscr{A}^{*}$ becomes a commutative Banach $\mathscr{A}$-bimodule. Moreover, $\mathscr{A}$ is Arens regular if and only if $\mathscr{A}^{* *}$ is commutative with respect to either, and hence both, of the Arens products.

We call $T \in \mathscr{A}^{*}$ weakly almost periodic if $\mathscr{O}(T)=\left\{u \cdot T \mid\|u\|_{\mathscr{A}} \leq 1\right\}$ is relatively weakly compact. $T$ is uniformly continuous if $T$ is in the norm closure of $\operatorname{span}\left\{u \cdot T_{1} \mid u \in \mathscr{A}, T_{1} \in \mathscr{A}^{*}\right\}$. When $\mathscr{A}=A_{p}(G)$, we denote the weakly almost periodic functionals by $W_{p}(\widehat{G})$ and the uniformly continuous functionals by $U C B_{p}(\widehat{G})$ (see [9]).

A locally compact group $G$ is amenable if there exists $m \in L_{\infty}(G)^{*}$ such that $m(1)=\|m\|=1$ and $m\left(L_{x} f\right)=m(f)$ where $L_{x} f(y)=$ $f\left(x^{-1} y\right)$ for every $x, y \in G$. The functional $m$ is called a left invariant mean on $L_{\infty}(G)$. All commutative locally compact groups and all: compact groups are amenable. $F_{2}$, the free group on two generators, is not amenable.

A functional $m \in P M_{p}(G)^{*}$ is called a topologically invariant mean on $P M_{p}(G)$ if $\|m\|=1$ and $m(u T)=u(e) m(T)$ for every $u \in A_{p}(G)$, 
$T \in P M_{p}(G)$. For any locally compact group it is known that $P M_{p}(G)$ has a T.I.M. [9, Proposition 2]. We can speak of a topologically invariant mean on any closed $A_{p}(G)$-submodule of $P M_{p}(G)$ which contains the functional $L_{e}\left(L_{e}(u)=u(e)\right)$. It is known that $W_{p}(\widehat{G})$ always has a unique invariant mean [9, Proposition 9].

A Banach space $X$ has the Radon-Nikodym Property of R.N.P. if every closed convex bounded subset $C \subseteq X$ is dentable. That is, for every $\varepsilon>0$ there exists $x \in C$ such that $x \notin \overline{c 0}\left\{C \backslash B_{\varepsilon}(x)\right\}$ where $B_{\varepsilon}(x)=\{y \in X \mid\|x-y\|<\varepsilon\}$. See [23, §2] for further information on the R.N.P.

3. Arens regularity. We begin with the following useful lemma.

LEMMA 3.1. Let $G$ be a locally compact group for which $A_{p}(G)$ is Arens regular. Then

(i) If $I$ is a closed ideal of $A_{p}(G)$, then $I$ is Arens regular.

(ii) If $H$ is a closed subgroup of $G$, then $A_{p}(H)$ is Arens regular.

(iii) If $K$ is a compact normal subgroup of $G$, then $A_{p}(G / K)$ is Arens regular.

Proof. (i) This follows from [4, p. 312, Corollary].

(ii) By appealing to [13] and by the following the arguments of [8, Lemma 3.8], we can show that $A_{p}(H)$ is isometrically isomorphic to $A_{p}(G) / I_{p}(H)$. Hence by $\left[4\right.$, p. 312 , Corollary], $A_{p}(G)$ is Arens regular.

(iii) $A_{p}(G / K)$ is isometrically isomorphic to the closed subalgebra of $A_{p}(G)$ consisting of functions which are constant on cosets of $K$ [13, Proposition 6]. The Arens regularity of $A_{p}(G / K)$ now follows immediately from $[4$, p. 312 , Corollary]

THEOREM 3.2. Let $G$ be a locally compact group for which $A_{p}(G)$ is Arens regular. Then $G$ is discrete.

Proof. We first assume that $G$ is separable. If $A_{p}(G)$ is Arens regular, then $W_{p}(\widehat{G})=P M_{p}(G)$. Hence $U C B_{p}(\widehat{G}) \subseteq W_{p}(\widehat{G})$ and $G$ is discrete by [9, Theorem 16].

Let $G$ be an arbitrary locally compact group. Let $U$ be an open neighborhood of $\{e\}$ in $G$ with compact closure. Then $U$ generates an open $\sigma$-compact subgroup $G_{0}$ of $G$. By Lemma (3.1)(ii), $A_{p}\left(G_{0}\right)$ is also Arens regular. Since $G_{0}$ is compactly generated either $G_{0}$ is 
discrete and we are done or there is a compact normal subgroup $K$ in $G_{0}$ such that $\lambda(K)=0$ and $G_{0} / K$ is separable.

Assume the latter to be true. By Lemma 3.1(ii), $A_{p}\left(G_{0} / K\right)$ is Arens regular. But $G_{0} / K$ is separable and therefore must be discrete. Thus $K$ is an open subgroup which contradicts the assumption that $\lambda(K)=0$. Hence $G_{0}$ must be discrete. Consequently, so must $G$ be discrete.

The next result generalizes [15, Theorem 3.7]. The proof is similar.

LemMA 3.3. Let $G$ be a locally compact group. Then $A_{p}(G)$ is an ideal in $P M_{p}(G)^{*}$ if and only if $G$ is discrete.

Proof. Assume first that $G$ is discrete. Then $U C B_{p}(G)=P F_{p}(G)$ [9, Proposition 15]. Let $u \in A_{p}(G)$ and $m \in P M_{p}(G)^{*}$. Let $T \in$ $P M_{p}(G)$. Then

$$
\begin{aligned}
\langle m \odot u, T\rangle & =\langle m, u T\rangle \\
& =\langle v, u T\rangle \quad \text { for some } v \in W_{p}(G)=P F_{p}(G)^{*} \\
& =\langle v u, T\rangle .
\end{aligned}
$$

Therefore $m \odot u=v u \in A_{p}(G)$. Also, $\langle u \odot m, T\rangle=\langle u, m \odot T\rangle=$ $\langle m, u T\rangle=\langle m \odot u, T\rangle$, so $A_{p}(G)$ is in the center of $P M_{p}(G)^{*}$. Therefore $A_{p}(G)$ is a closed two-sided ideal in $P M_{p}(G)^{*}$.

Conversely, assume that $A_{p}(G)$ is an ideal in $P M_{p}(G)^{*}$. Let $u_{0} \in$ $A_{p}(G)$ with $u(e)=1=\|u\|_{A_{p}(G)}$. Let

$$
K=\left\{m \odot u_{0} \mid m \in P M_{p}(G)^{*}, \quad m\left(L_{e}\right)=1=\|m\|\right\} .
$$

Since $m \mapsto m \odot u_{0}$ is weak-* to weak-* continuous $\left\{m \in P M_{p}(G)^{*} \mid\right.$ $\left.m\left(L_{e}\right)=1=\|m\|\right\}$ is weak-* compact, so is $K$. But $K \subset A_{p}(G)$, so $K$ is weakly compact in $A_{p}(G)$. It is also clearly convex.

For each $v \in A_{p}(G)$ with $v(e)=\|u\|_{A_{p}(G)}=1$, define the operator $\Gamma_{v}$ on $K$ by $\Gamma_{v}(w)=v w$, for every $w \in K$. The operators $\Gamma_{v}$ are pairwise commuting and $(K$, weak) to $(K$, weak) continuous. By the Kakutani-Markov fixed point theorem [8, p. 458], there exists some $v_{0} \in K$ such that $\Gamma_{v}\left(v_{0}\right)=v_{0}$ for every $v \in A_{p}(G)$ with $\|v\|_{A_{p}(G)}=v(e)=1$. That is, $v v_{0}=v_{0}$. Since $v_{0} \in K, v_{0}(e)=1$. Let $x \in G \sim\{e\}$. Then there exists $v_{1} \in A_{p}(G)$ with $v_{1}(e)=$ $1=\left\|v_{1}\right\|_{A_{p}(G)}$ while $v_{1}(x)=0$. Therefore $v_{0}(x)=v_{1}(x) v_{0}(x)=0$. Hence $v_{0}=1_{\{e\}}$ and $G$ is discrete. 
Proposition 3.4. Let $G$ be a locally compact group for which $A_{p}(G)$ is Arens regular. If $G$ is amenable, then $P M_{p}(G)$ has the RadonNikodym Property.

Proof. Since $A_{p}(G)$ is Arens regular, $A_{p}(G)$ is a two-sided ideal in its second dual. Since $G$ is amenable, $A_{p}(G)$ has a bounded approximate identity. It follows from [23, Corollary 3.2], that $P M_{p}(G)$ has the Radon-Nikodym Property.

Proposition 3.5. Let $G$ be a locally compact group for which $A_{p}(G)$ is Arens regular. Assume that $G$ is amenable. Then the following are equivalent:

(i) $A_{p}(G)$ is weakly sequentially complete,

(ii) $G$ is finite.

Proof. (i) $\rightarrow$ (ii). If $A_{p}(G)$ is weakly sequentially complete and $G$ is amenable, then by [23, Corollary 3.9] $A_{p}(G)$ is reflexive. It follows from [11, Theorem 4], that $A_{p}(G)$ is finite dimensional and hence $G$ is finite.

(ii) $\rightarrow$ (i). If $G$ is finite $A_{p}(G)$ is finite dimensional.

Corollary 3.6. Let $G$ be an amenable locally compact group. Then $A(G)$ is Arens regular if and only if $G$ is finite.

Proof. Since $A_{2}(G)=A(G)$ is the predual of a von Neumann algebra, it is weakly sequentially complete. If $A(G)$ is Arens regular, then $G$ is finite by Proposition 3.5.

Conversely, if $G$ is finite, then $A(G)$ is reflexive. Hence $A(G)^{* *}=$ $A(G)$ is commutative. Therefore $A(G)$ is Arens regular.

Corollary 3.6 is due to Lau and Wong [17]. They consider only the case of amenable groups where it is known that $W_{p}(\widehat{G}) \subseteq U C B_{p}(\widehat{G})[9$, Proposition 14]. For non-amenable groups, it is not known whether the above inclusion holds even for $p=2$ and for $G$ discrete. For $p=2$, the following proposition sheds some light on the non-amenable case.

Proposition 3.7. Let $G$ be a locally compact group for which $A(G)$ is Arens regular. Let $H$ be an amenable subgroup of $G$. Then $H$ is finite. In particular, $G$ is periodic.

Proof. By Lemma 3.1(ii), $A(H)$ is Arens regular. Hence by Corollary $3.6, H$ is finite. 
Let $x \in G$. Then $H=\langle x\rangle$, the subgroup generated by $x$ is commutative and hence amenable. Therefore $H$ is finite and $G$ is periodic.

COROLlARY 3.8. Let $G$ be a discrete group which contains the free group on 2 generators. Then $A(G)$ is not Arens regular.

One of the most famous conjectures in the study of amenable groups was that a discrete group $G$ would be amenable if and only if $G$ did not contain a subgroup isomorphic to the free group on 2 generators. $\mathrm{Ol}^{\prime}$ shanskii [18] has proved this conjecture to be false by constructing a non-amenable group $G$ for which every non-trivial proper subgroup is infinite cyclic. It follows from Proposition 3.7 that $A(G)$ is not Arens regular for this $G$. The natural question which arises is: Are there non-amenable periodic groups without infinite amenable subgroups?

Let $\mathscr{Z}$ be a class of groups such that if $G \in \mathscr{X}$, then any homomorphic image of $G$ also belongs to $\mathscr{X}$. A group $H$ is called a hyper- $\mathscr{X}$-group if every homomorphic image $H_{1} \neq\{e\}$ of $H$ has a normal $\mathscr{X}$-subgroup $N \neq\{e\}$.

Proposition 3.9. Let $G$ be a discrete group which satisfies any of the following conditions:

(i) $G$ is locally finite,

(ii) $G$ is isomorphic to a subgroup of $\operatorname{GL}(n, \mathbb{F})$ for some $n$ and any field $\mathbb{F}$,

(iii) $G$ is a 2-group,

(iv) $G$ is hyperfinite,

(v) $G$ has an involution $x$ with $\left|C_{G}(x)\right|<\infty$,

(vi) $G$ is hypercentral.

Then $A(G)$ is Arens regular if and only if $G$ is finite.

Proof. (i) If $G$ is locally finite, then every finitely generated subgroup is finite and hence amenable. Therefore $G$ is amenable [see 19, p. 121] and the result follows from Corollary 3.6.

(ii) If $A(G)$ is Arens regular, then $G$ is periodic. Hence $G$ is locally finite $[15$, p. 60$]$.

(iii) If $G$ is an infinite 2-group, then $G$ has an infinite abelian subgroup [15, p. 72]. Therefore $A(G)$ is not Arens regular.

(iv) Assume that $G$ is hyperfinite and that $A(G)$ is Arens regular. Then by (iii) every elementary abelian 2-subgroup is finite. Hence $G$ is finite $[15, \mathrm{p} .6]$. 
(v) If $G$ is infinite, then $[15,2.1$ Theorem] implies that $G$ contains an infinite abelian subgroup which is impossible.

(vi) If $G$ is hypercentral, then $G$ is locally nilpotent $[15$, p. 10] and hence amenable.

For $p \neq 2$, we are unable to show that $U C B_{p}(\widehat{G})=P M_{p}(G)$ implies that $G$ is compact. Though we believe this to be true, this still remains the main stumbling block preventing the extension of Corollary 3.6 for $p \neq 2$.

Proposition 3.10. Let $G$ be an amenable locally compact group. Then $G$ is discrete and $A_{p}(G)$ is Arens regular if and only if $P M_{p}(G)^{*}$ $=B_{p}(G)$.

Proof. Assume that $P M_{p}(G)^{*}=B_{p}(G)$. Then since $A_{p}(G)^{* *}$ is commutative, $A_{p}(G)$ is Arens regular. Hence $G$ is discrete, by Theorem 3.2.

Conversely, if $G$ is discrete, then $U C B_{p}(\widehat{G})=P F_{p}(G)$. If $G$ is Arens regular, then $P F_{p}(G)=P M_{p}(G)$. Hence $P M_{p}(G)^{*}=B_{p}(G)$ as a Banach space. Let $u, v \in B_{p}(G)$. Let $f \in l_{1}(G)$. Then $\langle u \odot v, f\rangle=$ $\langle u, v f\rangle=\int u v f d x=\langle u v, f\rangle$. Since $P M_{p}(G)=P F_{p}(G), l_{1}(G)$ is norm dense in $P M_{p}(G)$. Therefore $u \odot v=u v$ and the Arens multiplication agrees with the pointwise product on $B_{p}(G)$.

Proposition 3.11. Let $G$ be a countable amenable discrete group. If $A_{p}(G)$ is Arens regular, then $P_{p}(G)$ is separable.

Proof. By Proposition 3.4, $P M_{p}(G)$ has the R.N.P. However, since $G$ is countable, $A_{p}(G)$ is separable. It follows that $P M_{p}(G)$ is also separable [see $23, \S 2]$.

When $p=2, P M_{p}(G)$ is a von Neumann algebra. Since a separable von Neumann algebra is well known to be finite dimensional, we have another proof of Corollary 3.6. This follows since an infinite group must always have a countable infinite subgroup.

For amenable groups $P M_{p}(G)$ can be identified with the multipliers of $L_{p}(G)$, that is, the algebra of all operators on $L_{p}(G)$ which commute with convolution. The assumption of Arens regularity of $A_{p}(G)$ implies that the closure of $L_{1}(G)$ is the same with respect to both the norm topology and the weak operator topology on $B\left(L_{p}(G)\right)$. This would seem to suggest that $L_{p}(G)$ is finite dimensional and therefore 
that $G$ is finite. We are left to ponder the following two questions:

Problem 1. If $P_{p}(G)$ is separable for some $1<p<\infty$, is $G$ necessarily finite?

Problem 2. If $L_{1}(G)$ is norm dense in $P M_{p}(G)$, is $G$ necessarily finite?

Let $G$ be a discrete group. Let $\left\{x_{1}, \ldots, x_{n}\right\}=A$ be a finite subset of $G$. Then $I_{p}(G \backslash A)$ is a closed finite dimensional ideal in $A_{p}(G)$ and is therefore Arens regular. Moreover, $I_{p}(G \backslash A)$ has an identity.

Conversely, if a non-zero closed ideal in $A_{2}(G)$ is Arens regular, then this will be shown below to be sufficient to insure that $G$ is discrete. If, in addition, we assume that $I$ has a bounded approximate identity, then we will also show that $I$ is reflexive and therefore infinite codimensional.

THEOREM 3.12. Let $G$ be a locally compact group. Let $I$ be a closed non-zero ideal in $A_{p}(G)$. Assume that $I$ is Arens regular. Then $P M_{p}(G)$ has a unique topologically invariant mean.

Proof. Let $Z(I)=A \subset G$. Since $I$ is non-zero, $A \neq G$. Therefore $G \backslash A$ is open. By translating if necessary, we can assume that $G \backslash A$ is a neighborhood of $e$.

Let $M \in T I M_{p}(\widehat{G})$. Let $T \in I^{\perp}$. We can find $u \in A_{p}(G)$ such that $u \in I$ and $u(e)=1$. It follows that $\langle u T, v\rangle=\langle T, u v\rangle=0$ for every $v \in A(G)$. Hence $u T=0$. But then $0=m(u T)=u(e) m(T)=$ $m(T)$. Therefore $m \in I^{\perp \perp}$. Since we can identify $I^{\perp \perp}$ with $I^{* *}$, we have $T I M_{p}(\widehat{G}) \subseteq I^{* *}$.

Assume that $m_{1}, m_{2} \in T M_{p}(\widehat{G})$. It is easy to see that $m_{1} \odot m_{2}=$ $m_{1}$. In fact, given any $T \in P M_{p}(G)$ and any $u \in A_{p}(G)$, we have that $\left\langle T \odot m_{1}, u\right\rangle=\left\langle m_{1}, u T\right\rangle=u(e)\left\langle m_{1}, T\right\rangle$. Hence $T \odot m_{1}=\left\langle m_{1}, T\right\rangle L_{e}$. Finally, $\left\langle m_{1} \odot m_{2}, T\right\rangle=\left\langle m_{2}, T \odot m_{1}\right\rangle=\left\langle m_{1}, T\right\rangle\left\langle m_{2}, L_{e}\right\rangle=\left\langle m_{1}, T\right\rangle$. However, since $I$ is Arens regular, $I^{* *}$ is commutative. Therefore $m_{1}=m_{1} \odot m_{2}=m_{2} \odot m_{1}=m_{2}$.

COROLLARY 3.13. Let $G$ be a second countable locally compact group. Let I be a closed non-zero ideal in $A(G)$. If I is Arens regular; then $G$ is discrete.

Proof. By Theorem 3.12, $P M_{2}(G)$ has a unique topologically invariant mean. Consequently, $G$ is discrete [10, Theorem 1]. 
Proposition 3.14. Let I be a proper closed ideal in $A(G)$ with a bounded approximate identity. Then $I$ is Arens regular if and only if $I$ is reflexive.

Proof. A reflexive ideal is clearly Arens regular.

Conversely, assume that $I$ is Arens regular. Then $G$ is a discrete group. As $I$ has a bounded approximate identity, Cohen's Factorization Theorem [14, Corollary 32.26] implies that $I=I^{2}=\{u v \mid u, v \in$ $I\}$. Therefore $I \circ I^{* *}=(I \cdot I) \circ\left(I^{\perp \perp}\right) \subseteq I \circ\left(I \circ A(G)^{* *}\right) \subseteq I \cdot A(G) \subseteq I$. Hence $I$ is an ideal in $I^{* *}$. Also, since $A(G)$ is weakly sequentially complete, $I$ is weakly sequentially complete. It follows from [23, Corollary 3.7] and [23, Corollary 3.9] that $I$ is reflexive.

With Corollary 3.6 in mind, one might ask whether it is possible to have infinite dimensional ideals $I$ which are Arens regular or reflexive. In [11, Theorem 5], Granirer shows that while in a non-discrete group $A_{2}(G)$ has no non-zero reflexive ideals, (a fact that follows immediately from Corollary 3.13), every infinite discrete group is such that $A_{2}(G)$ contains an ideal isomorphic to $l_{2}$.

We close this section with some results on the Arens regularity of some related Banach algebras.

Proposition 3.15. Let $G$ be a locally compact group. Let $\mathscr{A}=$ $\left(B(G) \cap A P(G),\|\cdot\|_{B(G)}\right)$. Then $\mathscr{A}$ is Arens regular if and only if $A P(G)$ is finite dimensional.

Proof. $\mathscr{A}$ is isometrically isomorphic to $A\left(G^{\text {ap }}\right)$, where $G^{\text {ap }}$ denotes the almost periodic compactification of $G[7$, p. 203]. Since $G^{\text {ap }}$ is a compact group, it is amenable. Therefore $\mathscr{A}$ is Arens regular if and only if $G^{\text {ap }}$ is finite. But $G^{\text {ap }}$ is finite if and only if $A P(G)$ is finite dimensional.

The converse is obvious.

Corollary 3.16. Let $G$ be a locally compact group. If $B(G)$ is Arens regular, then $A P(G)$ is finite dimensional.

Observe that $A P(G) \cap B(G)$ is precisely the space of coefficient functions of the representation of $G$ obtained by lifting the left regular representation of $G^{\text {ap }}$ to $G$. In this case, the representation is such that its coefficient functions form an algebra. For a general representation $\pi$ of $G$, this is so if and only if $\pi \otimes \pi$ is quasi equivalent to a sub-representation of the representation $\pi$ [2, Proposition 3.26]. 
Assume that $G$ is a compact group. Let $\pi$ be a continuous unitary representation of $G$. Let $\mathscr{A}_{\pi}$ denote the closed self-adjoint subalgebra of $A(G)$ generated by the coefficients functions of $\pi$. Then we have the following result:

Proposition 3.17. Let $G$ be a compact group. Let $\pi$ be a continuous unitary representation of $G$. Then $A_{\pi}$ is Arens regular if and only if $\operatorname{ker} \pi$ is open.

Proof. $\mathscr{A}_{\pi}$ is isometrically isomorphic with $A(G / \operatorname{ker} \pi)$ [23]. Clear-: ly $G / \operatorname{ker} \pi$ is finite if and only if $\operatorname{ker} \pi$ is open. The result follows immediately from Corollary 3.6.

COROLlary 3.18. Let $G$ be compact and connected. Then $\mathscr{A}_{\pi}$ is Arens regular if and only if $\pi$ is the trivial representation.

We wish to bring the reader's attention to two related results in the literature which unfortunately contain errors. The first result is the equivalence of the unique invariant mean on $P M_{2}(G)$ with the discreteness of $G$. The proof of this result is usually attributed to Renaud [22]. However the proof of [22, Proposition 8] contains a serious error which may well be impossible to repair. It would therefore appear that at present the equivalence of the discreteness of $G$ with the existence of a unique invariant mean requires the assumption of second countability.

Secondly, in example 7.2 (b) of the deep paper [21], it is mistakenly stated that for every compact group $A_{p}(G)$ is Arens regular.

\section{REFERENCES}

[1] R. Arens, The adjoint of a bilinear operation, Proc. Amer. Math. Soc., 2 (1951), 839-848.

[2] G. Arsac, Sur l'espace de Banach engendré par les coefficients d'une représentation unitaire, Publ. Dép. Math. Lyon, 13 (1976), 1-101.

[3] P. Civin and B. Yood, The second conjugate space of a Banach algebra as an algebra, Pacific J. Math., 11 (1961), 847-870.

[4] J. Duncan and A. R. Hosseiniun, The second dual of a Banach algebra, Proc. Roy. Soc. Edinburgh, A84 (1979), 309-325.

[5] N. Dunford and J. T. Schwartz, Linear operators I, Interscience, New York (1958).

[6] C. F. Dunkl and D. E. Ramirez, Weakly almost periodic functions carried by hypercosets, Trans. Amer. Math. Soc., 185 (1972), 427-434.

[7] P. Eymard, L'algèbre de Fourier d'un groupe localement compact, Bull. Soc. Math. France, 92 (1964), 181-236. 
[8] B. Forrest, Amenability and bounded approximate identities in ideals in $A(G)$, Illinois J. Math., (1990), 1-25.

[9] E. Granirer, On some spaces of linear functionals on the algebras $A_{p}(G)$ for locally compact groups, Colloq. Math., LII (1987), 119-132.

[10] _ Properties of the set of topological invariant means on Eymard's $W^{*}$ algebra $V N(G)$, Indag. Math., 36 (1974), 116-121.

[11] _ On some properties of the Banach algebras $A_{p}(G)$ for locally compact groups, Proc. Amer. Math. Soc., 95 (1985), 375-381.

[12] E. Granirer and M. Leinert, On some topologies which coincide on the unit sphere of the Fourier-Stieltjes algebra $B(G)$ and the measure algebra $M(G)$, Rocky Mountain J. Math., 11 (1981), 459-472.

[13] C. Herz, Harmonic synthesis for subgroups, Ann. Inst. Fourier (Grenoble), 23 (1973), 91-123.

[14] E. Hewitt and K. A. Ross, Abstract Harmonic Analysis, vol II, Springer-Verlag, Berlin (1970).

[15] O. Kegel and B. Wehrfritz, Locally Finite Groups, North-Holland, Amsterdam (1973).

[16] A. T. Lau, The second conjugate algebra of the Fourier algebra of a locally compact group, Trans. Amer. Math. Soc., 267 (1981), 53-63.

[17] A. T. Lau and J. Wong, Weakly almost periodic elements in $L_{\infty}(G)$ of a locally compact group, Proc. Amer. Math. Soc., 107 (1989), 1031-1036.

[18] A. Yu. Ol'shanskii, An infinite simple Noetherian group without torsion, Math. U.S.S.R.-Izv., 15 (1980), 531-588.

[19] A. L. T. Paterson, Amenability, Math. Surveys, vol. 29, Amer. Math. Soc., Providence, R.I., (1988).

[20] J. P. Pier, Amenable Locally Compact Groups, Wiley, New York (1984).

[21] J. S. Pym and E. O. Oshobi, Banach algebras whose duals consist of multipliers, Math Proc. Cambridge Phil. Soc., 102 (1987), 481-505.

[22] P. F. Renaud, Invariant means on a class of von Neumann algebras, Trans. Amer. Math. Soc., 170 (1972), 495-498.

[23] A. Ülger, Arens regularity sometimes implies R.N.P., Pacific J. Math., 143 (1990), 377-399.

Received July 12, 1990 and in revised form November 2, 1990.

UNIVERSITY OF WATERLOO

WATERloo, OnTARIo, Canada 



\section{PACIFIC JOURNAL OF MATHEMATICS EDITORS}

\author{
V. S. VARADARAJAN \\ (Managing Editor) \\ University of California \\ Los Angeles, CA 90024-1555-05 \\ Herbert Clemens \\ University of Utah \\ Salt Lake City, UT 84112 \\ THOMAS ENRIGHT \\ University of California, San Diego \\ La Jolla, CA 92093
}

Nicholas ERcolani

University of Arizona

Tucson, AZ 85721

R. FINN

Stanford University

Stanford, CA 94305

VAUGHAN F. R. JONES

University of California

Berkeley, CA 94720

STEVEN KeRCKHOFF

Stanford University

Stanford, CA 94305

\section{C. MOORE \\ University of California \\ Berkeley, CA 94720}

MARTIN SChaRLEMANN

University of California

Santa Barbara, CA 93106

HAROLd STARK

University of California, San Diego

La Jolla, CA 92093

\section{ASSOCIATE EDITORS}

\begin{tabular}{|c|c|c|c|c|}
\hline ARENS & $\begin{array}{l}\text { E. F. BECKENBACH } \\
(1906-1982)\end{array}$ & NeumanN & $\begin{array}{l}\text { F. Wolf } \\
(1904-1989)\end{array}$ & K. Yoshida \\
\hline \multicolumn{5}{|c|}{ SUPPORTING INSTITUTIONS } \\
\hline \multirow{2}{*}{\multicolumn{2}{|c|}{$\begin{array}{l}\text { IVERSITY OF ARIZONA } \\
\text { IIVERSITY OF BRITISH COLUMBIA }\end{array}$}} & UNIVERS & Y OF OREGON & \\
\hline & & UNIVERS & OF SOUTHER & IIA \\
\hline \multicolumn{2}{|c|}{ LIFORNIA INSTITUTE OF TECHNOLOGY } & STANFO & UNIVER & \\
\hline \multirow{2}{*}{\multicolumn{2}{|c|}{$\begin{array}{l}\text { IVERSITY OF CALIFORNIA } \\
\text { ONTANA STATE UNIVERSITY }\end{array}$}} & UNIVERS & OF HAWAII & \\
\hline \multirow{2}{*}{\multicolumn{2}{|c|}{$\begin{array}{l}\text { ONTANA STATE UNIVERSITY } \\
\text { IIVERSITY OF NEVADA, RENO }\end{array}$}} & UNIVERS & YF TOKYO & \\
\hline & & UNIVERS & I OF UTAH & \\
\hline \multirow{2}{*}{\multicolumn{2}{|c|}{$\begin{array}{l}\text { W MEXICO STATE UNIVERSITY } \\
\text { EGON STATE UNIVERSITY }\end{array}$}} & WASHING & N STATE UNI & SITY \\
\hline & & UNIVERS & NASI & \\
\hline
\end{tabular}

The Supporting Institutions listed above contribute to the cost of publication of this Journal, but they are not owners or publishers and have no responsibility for its content or policies.

Mathematical papers intended for publication in the Pacific Journal of Mathematics should be in typed form or offset-reproduced (not dittoed), double spaced with large margins. Please do not use built up fractions in the text of the manuscript. However, you may use them in the displayed equations. Underline Greek letters in red, German in green, and script in blue. The first paragraph must be capable of being used separately as a synopsis of the entire paper. In particular it should contain no bibliographic references. Please propose a heading for the odd numbered pages of less than 35 characters. Manuscripts, in triplicate, may be sent to any one of the editors. Please classify according to the 1991 Mathematics Subject Classification scheme which can be found in the December index volumes of Mathematical Reviews. Supply name and address of author to whom proofs should be sent. All other communications should be addressed to the managing editor, or Elaine Barth, University of California, Los Angeles, California 90024-1555-05.

There are page-charges associated with articles appearing in the Pacific Journal of Mathematics. These charges are expected to be paid by the author's University, Government Agency or Company. If the author or authors do not have access to such Institutional support these charges are waived. Single authors will receive 50 free reprints; joint authors will receive a total of 100 free reprints. Additional copies may be obtained at cost in multiples of 50 .

The Pacific Journal of Mathematics (ISSN 0030-8730) is published monthly except for July and August. Regular subscription rate: $\$ 190.00$ a year (10 issues). Special rate: $\$ 95.00$ a year to individual members of supporting institutions.

Subscriptions, orders for numbers issued in the last three calendar years, and changes of address should be sent to Pacific Journal of Mathematics, P.O. Box 969, Carmel Valley, CA 93924, U.S.A. Old back numbers obtainable from Kraus Periodicals Co., Route 100, Millwood, NY 10546.

The Pacific Journal of Mathematics at P.O. Box 969, Carmel Valley, CA 93924 (ISSN 0030-8730) is published monthly except for July and August. Second-class postage paid at Carmel Valley, Californiä 93924, and additional mailing offices. Postmaster: send address changes to Pacific Journal of Mathematics, P.O. Box 969, Carmel Valley, CA 93924.

\section{PUBLISHED BY PACIFIC JOURNAL OF MATHEMATICS, A NON-PROFIT CORPORATION}




\section{Pacific Journal of Mathematics}

\section{Vol. 151, No. $2 \quad$ December, 1991}

Michael G. Eastwood and A. M. Pilato, On the density of twistor

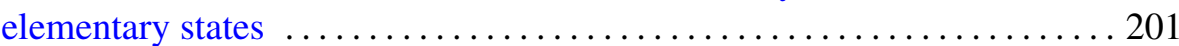

Brian E. Forrest, Arens regularity and discrete groups $\ldots \ldots \ldots \ldots \ldots \ldots 217$

Yu Li Fu, On Lipschitz stability for F.D.E ..................... 229

Douglas Austin Hensley, The largest digit in the continued fraction expansion of a rational number $\ldots \ldots \ldots \ldots \ldots \ldots \ldots \ldots \ldots \ldots \ldots . \ldots 237$

Uwe Kaiser, Link homotopy in $\mathbb{R}^{3}$ and $S^{3}$

Ronald Leslie Lipsman, The Penney-Fujiwara Plancherel formula for abelian symmetric spaces and completely solvable homogeneous

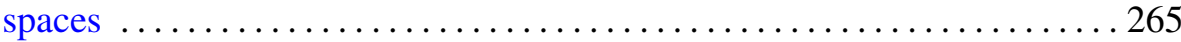

Florin G. Radulescu, Singularity of the radial subalgebra of $\mathscr{L}\left(F_{N}\right)$ and the

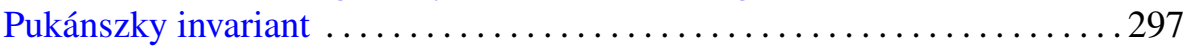

Albert Jeu-Liang Sheu, The structure of twisted SU(3) groups $\ldots . \ldots \ldots . \ldots 307$

Morwen Thistlethwaite, On the algebraic part of an alternating link . . . . . 317

Thomas (Toma) V. Tonev, Multi-tuple hulls .................. 335

Arno van den Essen, A note on Meisters and Olech's proof of the global asymptotic stability Jacobian conjecture ..................... 351

Hendrik J. van Maldeghem, A characterization of the finite Moufang hexagons by generalized homologies

Bun Wong, A note on homotopy complex surfaces with negative tangent bundles

Chung-Tao Yang, Any Blaschke manifold of the homotopy type of $\mathbf{C} P^{n}$ has the right volume 